\title{
The Electrodynamics of Charged Dust in the Cometary Environment
}

\author{
M. HORANYI \\ Lunar and Planetary Laboratory \\ Department of Planetary Sciences \\ University of Arizona \\ Tucson, Arizona 85721
}

\author{
D.A. MENDIS \\ Department of Electrical and \\ Computer Engineering \\ University of California, San Diego \\ La Jolla, CA 92093
}

\begin{abstract}
Dust in the plasma and radiative environment of a comet is necessarily electrically charged. This charging has both physical and dynamical effects on the dust, being particularly important on the smallest particles observed in the dust size spectrum. In this paper, these dynamical effects are reviewed and the pertinent observations are discussed.
\end{abstract}

\section{Introduction}

The systematic study of the dynamics of the dust tails of comets was stimulated by the 1835 apparition of Halley's comet. In an attempt to explain the morphology of the dust tail, Bessel (1836) derived the equations of motion of dust particles emitted from the nucleus and driven away from the Sun by some unknown repulsive force, which, like solar gravitation, varied inversely with heliocentric distance. The physical nature of this postulated repulsive force was recognized only much later to be solar radiation pressure (Arrhenius, 1900; Schwarzchild, 1901).

While significant contributions to dust tail dynamics were subsequently made by a number of authors, it was brought to fruition by Finson and Probstein (1968 a,b). Using a synthetic approach that combined the earlier concepts of syndynes (the loci in the cometocentric frame connecting particles having the same ratio of the solar radiation force to solar gravity) and synchrones (the loci in the cometocentric frame connecting particles emitted from the comet at the same time), Finson and Probstein inferred a number of cometary dust parameters, such as the size distribution weighted by the light scattering efficiency of the particles, by comparing calculated and observed isophotes of the tail. This method, with few modifications, has been the basis of all subsequent studies of cometary dust dynamics. Probstein (1968) was also the first to study in detail the problem of dust-gas coupling in the inner coma. This led to the realization that while the dust-gas coupling was confined to a thin region ( $\left.\leq 10 R_{n}\right)$ surrounding the nucleus, the drag of the dust on the expanding gas made its flow transonic.

These studies have been extensively reviewed elsewhere (e.g., see Mendis et al., 1985 ) and will not be further pursued in this brief review. Here we will concentrate on an aspect of cometary dust dynamics that has not been the subject of extensive study until rather recently, but whose importance is underscored by the discovery of very small $\left(\mathrm{m}_{\mathrm{d}} \sim 10^{-15}\right.$ to $\left.3 \times 10^{-17} \mathrm{~g}\right)$ dust particles in the environment of Halley's comet. This is the effect of electrodynamic forces on dust grains (which must necessarily be charged in the plasma and radiative environment of the comet) as they move through the magnetoplasma environment of the comet. 


\section{The Role of Radiation Pressure}

Once a dust grain (which is assumed here to be uncharged) leaves the region of dust-gas interaction, the only two forces acting on it in the heliocentric frame are the solar radiation (pressure) force $F_{\text {rad }}$ and solar gravity $F_{\text {grav }}$, since the gravitational force of the cometary nucleus itself is negligible. Since

$$
\mathrm{F}_{\mathrm{rad}}=\frac{\mathrm{Q}_{\mathrm{pr}}}{\mathrm{c}}\left(\frac{\mathrm{L}_{\odot}}{4 \pi \mathrm{r}^{2}}\right) \cdot \pi \mathrm{a}^{2}
$$

and

$$
\mathrm{F}_{\text {grav }}=\frac{\mathrm{GM}_{\odot}}{\mathrm{r}^{2}} \cdot \frac{4}{3} \pi \mathrm{a}^{3} \rho_{\mathrm{d}}
$$

where $\mathrm{L}_{\odot}$ is the mean solar luminosity $\left(=3.90 \times 10^{33} \mathrm{ergs}^{-1}\right)$, $\mathrm{Q}_{\mathrm{pr}}$ is the scattering efficiency for solar radiation pressure and the other quantities have their usual meaning,

$$
\frac{\mathrm{F}_{\mathrm{rad}}}{\mathrm{F}_{\mathrm{grav}}} \simeq \frac{5.95 \times 10^{-5} \mathrm{Q}_{\mathrm{pr}}}{\rho_{\mathrm{d}} \cdot \mathrm{a}}
$$

Both for dielectrics (e.g., olivine) and for conductors (e.g. magnetite) $\mathrm{Q}_{\mathrm{pr}}$ remains roughly constant for $2 \times 10^{-5} \mathrm{~cm} \leq \mathrm{a} \leq 10^{-2} \mathrm{~cm}$ and declines steeply for $\mathrm{a} \leq 2 \times 10^{-5} \mathrm{~cm}$, with the decline being much steeper for dielectrics. Typically, in the range 2 $\times 10^{-5} \mathrm{~cm} \leq \mathrm{a} \leq 10^{-2} \mathrm{~cm}, \mathrm{Q}_{\mathrm{pr}} \geq 0.5$ for dielectrics, and $\mathrm{Q}_{\mathrm{pr}} \geq 1.0$ for conductors. Consequently, $\mathrm{F}_{\text {rad }} \approx \mathrm{F}_{\text {grav }}$ when $\mathrm{a} \approx 0.3 \mu \mathrm{m}$ and $\mathrm{a} \approx 0.6 \mu \mathrm{m}$, respectively, in the two cases.

For typical times of flight $\leq 5 \times 10^{5} \mathrm{~s}$, the cometocentric frame can be considered to be an inertial one. In this frame, the only force acting on the dust particle is the radiation pressure directed along the Sun-comet line, and this force is approximately a constant for a grain of given size. Consequently, grains of a given size that are emitted at various angles to the Sun-comet axis with initial speed $v_{i}(=$ the terminal speed acquired due to the gas drag) will move in parabolic orbits, all of which are enveloped within a paraboloid of revolution whose apex distance, $\alpha$ is equal to $v_{i}^{2} / 2 \mathrm{~g}$, with $\mathrm{g}=\mathrm{F}_{\mathrm{rad}} / \mathrm{m}_{\mathrm{d}}$ (see Figure 1).

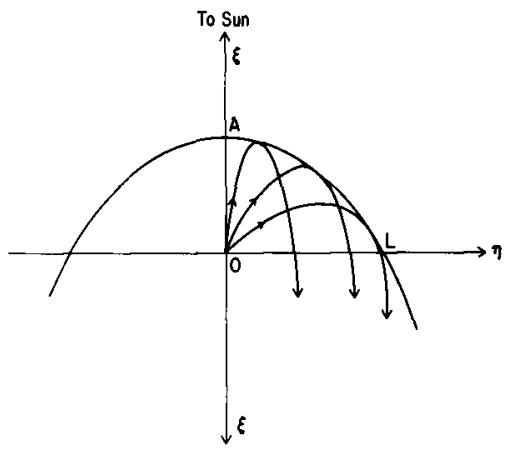

Fig. 1. The parabolic trajectories and the enveloping paraboloid for unchanged dust grains in a plane containing the Sun-comet axis. 
Using values of $v_{i}$ calculated by Gombosi and Horanyi (1986) and values of $Q_{p r}$ for olivine and magnetite obtained from Divine (1984), the values of the apex distance, $\alpha$, for different size grains are shown in Figure 2.

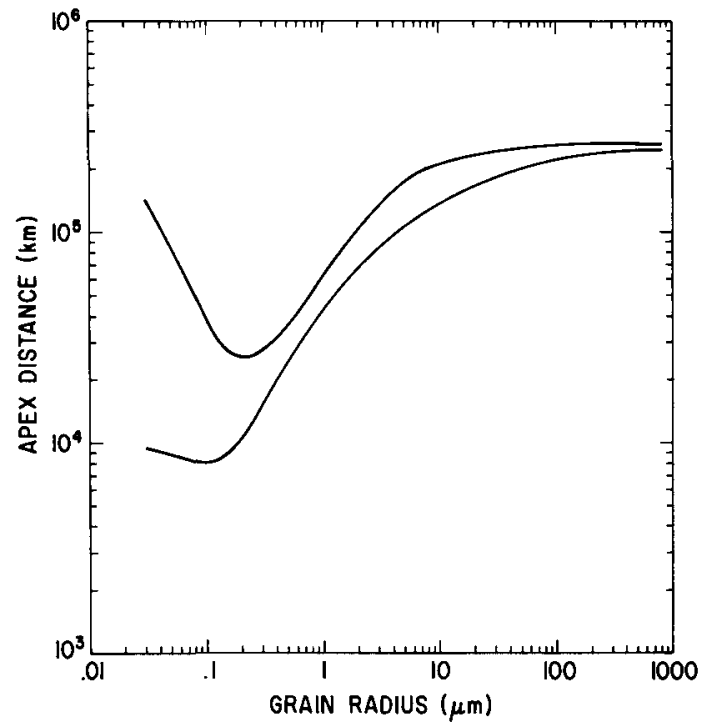

Fig. 2. The apex distance of the bounding paraboloid for uncharged olivine (upper curve) and magnetite (lower curve) grains as a function of size.

\section{Consequences of Grain Charging}

Contrary to the assumption of the preceeding section, it is now generally recognized that dust grains in the cometary environment are necessarily electrically charged. This electrostatic charging of dust grains in the cometary environment was discussed by Notni (1966), but the question did not receive further attention for some time.

Cometary dust, in common with much of cosmic dust, is immersed in an ultraviolet-radiative and plasma environment. The dust grains are thus subject to various fluxes of electric charge (currents), which proceed to charge up the grains to some electrostatic potential, $\phi_{g}$, with respect to that of the ambient plasma, $\bar{\phi}$. The basic equation governing the grain charging is:

$$
\frac{\mathrm{dQ}}{\mathrm{dt}}=\mathrm{I} ; \mathrm{Q}=\mathrm{C}\left(\phi_{\mathrm{g}}-\bar{\phi}\right)
$$

where $Q$ is the grain charge, $C$ is the grain capacitance, and $I$ is the net current onto the grain. While I depends on the physical and electrical properties of the grain, environmental conditions, $\Delta \phi$, and the relative velocity between the grain and the plasma, contributions to it come from several sources. The most important in the cometary environment are electron and ion fluxes, photoemission induced by UV radiation, and secondary emission of electrons by the grain due to energetic electron impact in regions where the electron energy is sufficiently high. (For a detailed general discussion of these, as well as of other charging currents that arise in different environments, see, e.g., Whipple (1981).) 
Using the insitu dust and plasma data at Halley's comet, it is easy to see that average intergrain distances $d$, even for the smallest grains observed, is considerably larger than the Debye shielding distances $(\lambda)$. Consequently, the grains may be considered as isolated, so that we may regard $\bar{\phi} \approx 0$ and $C=C_{\text {iso }}=a\left(1+\frac{a}{\lambda}\right) \simeq a$ (in c.g.s. units) for small grains since $\lambda \geqslant 5 \mathrm{~cm}$.

When $\frac{\mathrm{dQ}}{\mathrm{dt}}=0$, solution of (4) leads to the equilibrium potential $\phi_{\mathrm{eq}}$.

A recent calculation (Notni and Tiersch, 1987) of the variation of $\phi_{\mathrm{eq}}$ for three different types of grains (silicate, graphite, and aluminium) for different plasma compositions (pure $\mathrm{H}^{+}$and pure $\mathrm{H}_{2} \mathrm{O}^{+}$), plasma temperatures, and densities is shown in Figure 3.

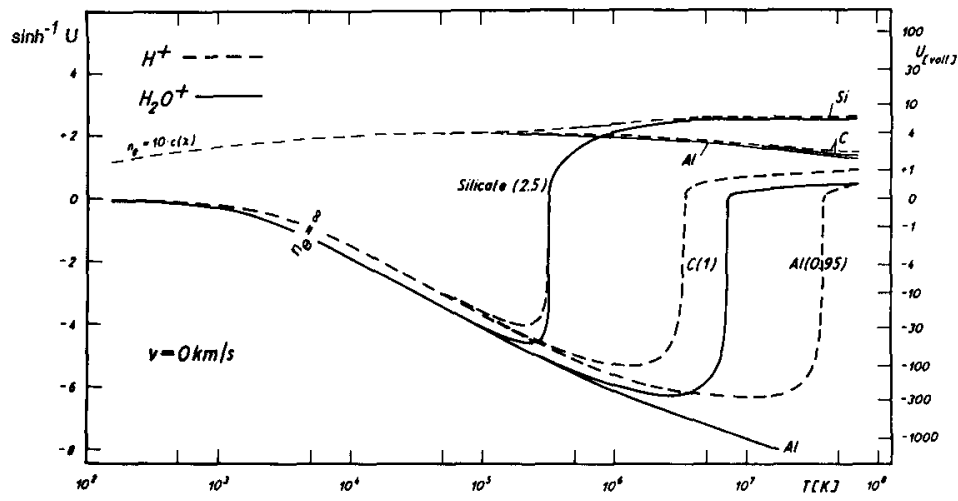

Fig. 3. The equilibrium potentials of grains of different compositions in dense and low density $\left(\mathrm{H}^{+}\right.$and $\mathrm{H}_{2} \mathrm{O}^{+}$) plasmas as a function of the plasma temperature. The grains are assumed to be stationary with respect to the plasma (from Notni and Tiersch, 1987).

The numbers within parenthesis are the maximum secondary emission yields for the appropriate material. In this calculation, the plasma energy $\left(\mathrm{kT}_{\mathrm{e}}\right)$, corresponding to the maximum secondary emission yield $\mathrm{E}_{\mathrm{m}}$ for silicate, graphite, and aluminium, is taken as $420 \mathrm{eV}, 250 \mathrm{eV}$, and $420 \mathrm{eV}$, respectively. Only two values of the electron density are used; very large $\left(n_{e} \simeq \infty\right)$ and $n_{e}=10 c(x)$, where $c(x)$ is the photoemission yield coefficient.

It is seen that in the region of relatively high plasma densities (where photoemission is unimportant) and relatively low temperatures $\left(\mathrm{k} \mathrm{T}_{\mathrm{e}} \leqslant 30 \mathrm{eV}\right.$, where secondary emission is unimportant), the grain potentials are negative and numerically small. Typically, $\mathrm{U} \simeq$ $2.5\left(\mathrm{kT}_{\mathrm{c}} / \mathrm{e}\right)$ volts for $\mathrm{H}^{+}$plasma and $\mathrm{U}=-4.0\left(\mathrm{kT}_{\mathrm{c}} / \mathrm{e}\right)$ volts for $\mathrm{H}_{2} \mathrm{O}^{+}$plasma. At higher temperatures, as secondary emission becomes important, the grain potential is seen to change from negative to positive, particularly for the high-yield silicate. Finally, it is observed that when photoemission is important (the uppermost curve), the grain potentials are positive. 
The rather uncertain secondary emission yield function adopted by these authors and others vary: Consequently, the calculated plasma temperature at which the transition from negative to positive potential takes place, as well as the maximum positive potential achieved, is different. However, the basic nature of the potential curves is similar to that of the curves exhibited in Figure 3.

Inside the dense cometary ionopause where $T_{e} \leq 10^{3 \circ} \mathrm{K}$ and $n_{e} \geq 10^{3} \mathrm{~cm}^{-3}, U \leq 0.2 \mathrm{~V}$. In the region of the largely undisturbed solar wind where photoemission is dominant, $\mathrm{U} \simeq$ 2 to $5 \mathrm{~V}$, whereas in the region between the outer shock and the ionopause, the grain potentials can assume both positive and negative values. While the potential of conducting grains (e.g., C) remain negative throughout $(\sim-10 \mathrm{~V}$ to $\sim-20 \mathrm{~V})$, those of dielectric grains (e.g., silicates) could switch, due to the importance of secondary emission.

The importance of electrical charging on the dynamics of the grains was discussed by Wallis and Hassan (1983), who pointed out that these changed grains are subject to electromagnetic forces due to the convectional electric fields both in the undisturbed solar wind and in the region of the shocked solar wind outside the ionopause.

The electrostatic acceleration of the grain, $\overrightarrow{\mathrm{g}}_{\mathrm{e}}$, is given by

$$
\overrightarrow{\mathrm{g}}_{\mathrm{e}}=\mathrm{Q} \frac{\overrightarrow{\mathrm{E}}}{\mathrm{m}_{\mathrm{d}}}=-\frac{\mathrm{Q} \overrightarrow{\mathrm{V}}_{\mathrm{sw}} \times \overrightarrow{\mathrm{B}}}{\mathrm{c} \mathrm{m}_{\mathrm{d}}}
$$

since $\left|\overrightarrow{\mathrm{V}}_{\mathrm{d}}\right| \ll\left|\overrightarrow{\mathrm{v}}_{\mathrm{sw}}\right|$.

To a good approximation, $\overrightarrow{\mid E} \mid$ is constant outside the ionopause along the Suncomet axis. Assuming that $\overrightarrow{\mid \mathrm{B}} \mid=5 \gamma$ and that $\overrightarrow{\mathrm{B}}$ is inclined at $45^{\circ}$ to $\overrightarrow{\mathrm{V}}_{\mathrm{sw}}$ at $1 \mathrm{AU}$, one obtains

$$
X=\frac{\vec{g}_{\mathrm{e}} \mid}{\left|\overrightarrow{\mathrm{g}}_{\mathrm{r}}\right|}=\frac{10^{-2}|\phi(\mathrm{V})|}{\mathrm{a}(\mu) \mathrm{Q}_{\mathrm{pr}}}
$$

The values of $X$ for olivine and magnetite grains of different sizes are given in Table 1, taking $|\phi(v)|=5$. It is seen that while $X \ll 1$ when $\mathrm{a}=1 \mu, \mathrm{X}=1$ when $\mathrm{a}=0.1 \mu$ for olivine. When $\mathrm{a}=0.03 \mu, \mathrm{X}>1$ for magnetite and $\mathrm{X}>1$ for olivine.

TABLE 1. The Ratio $X$ for Different Sized Grains

\begin{tabular}{l|c|c}
\hline \multirow{2}{*}{$\mathrm{a}(\mu)$} & \multicolumn{2}{|c}{$\mathrm{X}$} \\
\cline { 2 - 3 } & Olivine & Magnetite \\
\hline 1.0 & 0.05 & 0.04 \\
0.5 & 0.85 & 0.06 \\
0.1 & 1.00 & 0.28 \\
0.03 & 16.5 & 3.35 \\
\hline
\end{tabular}


The grains are now subject to the total acceleration $\vec{g}_{\mathrm{r}}+\overrightarrow{\mathrm{g}}_{\mathrm{e}}$. Assuming that $\overrightarrow{\mathrm{g}}_{\mathrm{e}}$ is also constant, it is obvious that dust particles emitted isotropically with initial speed $v_{i}$ will once again describe parabolic orbits that are enveloped by a paraboloid whose axis is now in the direction of $\overrightarrow{\mathrm{g}}_{\mathrm{r}}+\overrightarrow{\mathrm{g}}_{\mathrm{e}}$, as shown in Figure 4. Clearly, the axis would be inclined to the solar direction by a large angle for the smaller grains, as pointed out by Wallis and Hassan (1983). For example, for a $0.1-\mu$ olivine grain $\left(Q_{p r} \approx 0.5\right)$, if $|\phi|=5 \mathrm{~V}$, the angle, $\theta$, of the paraboloid axis to the solar direction $=45^{\circ}$, whereas it is still about $10^{\circ}$ even when $\mathrm{a} \simeq 0.5 \mu$ for olivine.

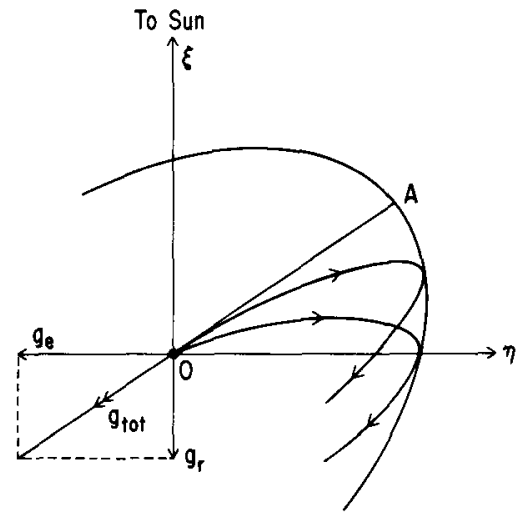

Fig. 4. The parabolic trajectories and the enveloping parabola of charged grains in the plane containing the Sun-comet axis and the convectional electric field vector in the cometocentric frame.

Of course, the smallest grains would not contribute appreciably to the solar radiation scattered by the cometary dust, and consequently their bounding envelopes may not be apparent in the visual. This is not the case for grains with radius $\simeq 0.5 \mu$ and if they are sufficiently charged $(|\phi|-5 \mathrm{~V})$ and are basically dielectric, their bounding envelopes should have axes inclined to the Sun-comet line at an angle that is apparent in visual observations. Indeed, this seems to be the case in some drawings of the sunward envelopes of comet Donati (1858 VI), as seen in Figure 5. So while it is tempting to interpret the skewness of these sunward envelopes as a consequence of the non-radial acceleration of charged dust grains, it must be pointed out that an alternative explanation has been proposed by Sekanina (1987), who interprets the skewness of these sunward envelopes in terms of spiral loci of particles emitted from localized regions in a rotating cometary nucleus.

In this connection, it is interesting to point out that the apex distance of the bounding paraboloid of charged grains is given by

$$
\alpha=\frac{v_{i}^{2}}{2 g_{t o t}}=\frac{v_{i}^{2}}{2 \sqrt{g_{r}^{2}+g_{e}^{2}}}
$$




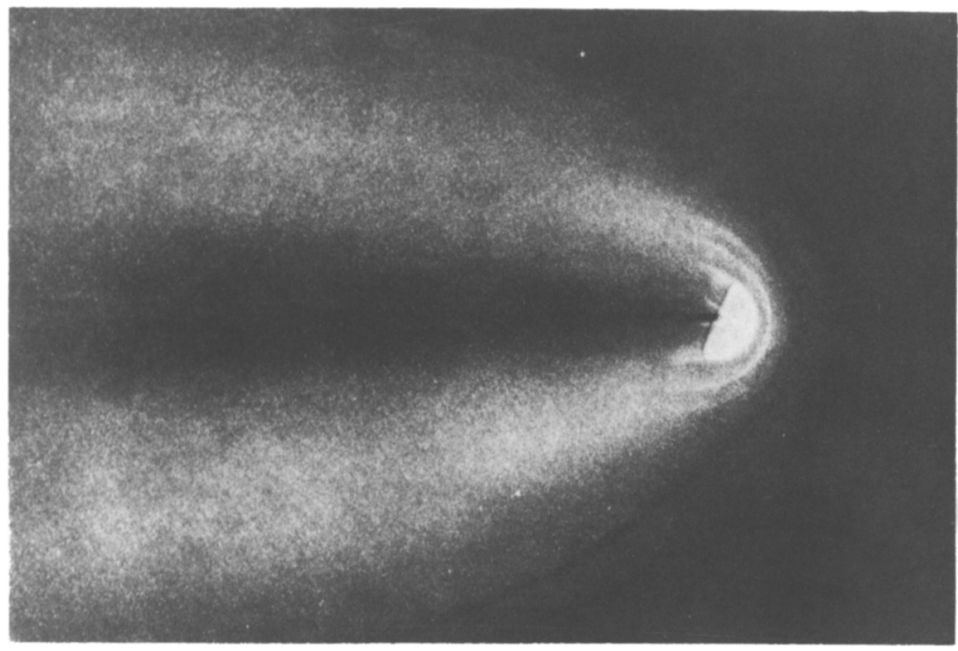

Fig. 5. Drawing based on visual observations of comet Donati 1858 VI by G.P. Bond on Oct. 9, 1858. The Sun-comet axis is given by the prolonged tail axis (from Rahe et al., 1969).

So while the grains are even more tightly constrained in the $\eta$-direction (see Figure 4) than if they were uncharged, they can move to greater distances from the Sun-comet line in the opposite sense. This could be a possible explanation for the detection of very small $\left(3 \times 10^{-17} \mathrm{~g} \leq \mathrm{md} \leq 10^{-14} \mathrm{~g}\right)$ grains by Vega 1 and Vega 2 inbound at distances as large as $2.5 \times 10^{5} \mathrm{~km}$ and $3.2 \times 10^{5} \mathrm{~km}$ from the nucleus of comet Halley (Vaisberg et al., 1987). Of course, the optical properties of these very small grains are unknown. However, if they are sufficiently absorbing (e.g., "dirty" silicates), as deduced from the infrared thermal emissions from several comets (Hanner, 1980), then the excursions of even $0.05-\mu \mathrm{m}$ grains from the nucleus in the directions of the encounters are likely to be $\leqslant 10^{5} \mathrm{~km}$.

\section{The Dynamics of Charged Dust}

The basic equation governing the dynamics of a charged dust grain in the cometocentric frame, regarded as an inertial frame is given by

$$
m_{d} \frac{d \vec{v}_{d}}{d t}=Q(t)\left[\vec{E}+\frac{\vec{v}_{d} \times \vec{B}}{c}\right]+\vec{F}_{r a d}+\vec{F}_{c}+\vec{F}_{i g}
$$

where $\vec{E}$ is the convectional electric field $(=-\vec{v} \times \vec{B} / c) ; \vec{v}$ is the plasma velocity; $\vec{F}_{r a d}, \vec{F}_{c}$ and $\overrightarrow{\mathrm{F}}_{\mathrm{ig}}$ are the radiative pressure force, the Coulomb drag, and the intergrain force, respectively; and $Q(t)$ is the grain charge, which is given by equation (4). In those instances where the cometocentric frame cannot be considered as an inertial frame, the appropriate inertial forces need to be included on the right-hand side of equation (8). In situations where the intergrain distance $d>\lambda$, which is generally true at comets, $F_{i g}$ may be neglected. 
In order to calculate the grain orbits, one needs $T_{e}(r), T_{i}(r), n_{e}(r), \vec{v}(\vec{r})$ and $\vec{B}(\vec{r})$, with the last two quantities giving $\vec{E}(\vec{r})$. Clearly, a detailed model of the plasma flow and the magnetic field in the cometary environment, such as the MHD model of Schmidt and Wegmann (1982), is needed. Horanyi and Mendis (1985) used a simpler approach. They used a simple 'source-in-a-uniform-stream' model to reproduce the stream lines (see Figure 6), then they proceeded to calculate the $\vec{E}$-field, noting that the stream-lines were also electric equipotentials in ideal MHD when $\vec{\nabla}$ and $\vec{B}$ are mutually perpendicular.

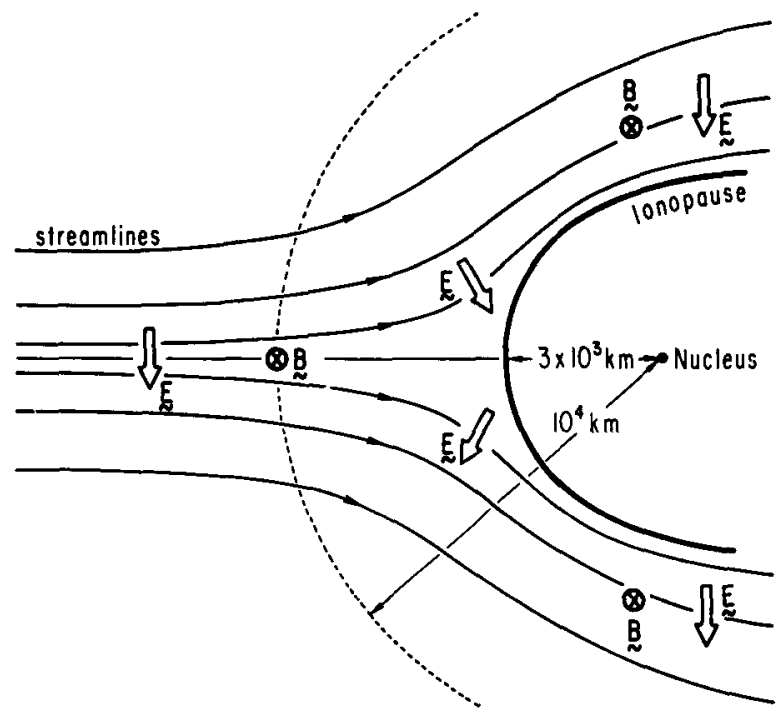

Fig. 6. Schematic of the plasma stream-lines and the convectional electric field in the vicinity of the ionopause. The magnetic field is assumed to be directed into the paper (from Horanyi and Mendis, 1985).

Horanyi and Mendis (1986 a,b) subsequently performed numerical simulations to calculate the distribution of charged dust in the environments of comets Halley and Giacobini-Zinner, taking into account the orbital motions of the comet. In the latter case, a scatter plot of the distribution of grains of various sizes in a plane normal to the Suncomet axis at a distance $10^{4} \mathrm{~km}$ behind the nucleus is shown in Figure 7. In this case, the interplanetary magnetic field is assumed to be in the orbital plane of the comet, so that the convectional electric field is normal to this plane.

The grain sizes increase from $0.03 \mu \mathrm{m}$ at the top of the figure to $1.0 \mu \mathrm{m}$ at the bottom. The first column shows the distributions when the electromagnetic effects are taken into account $\overrightarrow{(B} \neq \overrightarrow{0})$, while the second column shows the corresponding distributions when the electromagnetic effects are neglected (e.g., $\vec{B}=\overrightarrow{0}$ ). The two panels on the right are histograms of the column densities (in arbitrary units) corresponding to the distributions on the left. The third column corresponds to the case $B \neq 0$, while the last column corresponds to the case $\vec{B}=\overrightarrow{0}$. The nucleus in each plot is located at the origin of coordinates. The elongation of the distribution normal to the orbital plane is obvious, particu- 
larly for the smallest grains $(0.03 \mu \mathrm{m})$. In that case, it is also seen that the grains are concentrated well below the orbital plane. The larger grains have a more symmetrical distribution about the axis. In the absence of electromagnetic effects (e.g., $\vec{B}=\overrightarrow{0}$ ), it is seen, once more, that the larger ("older") grains concentrate away from the axis, while the smaller ("younger") grains concentrate closer to the axis. These distributions are, of course, axially symmetric in this case.
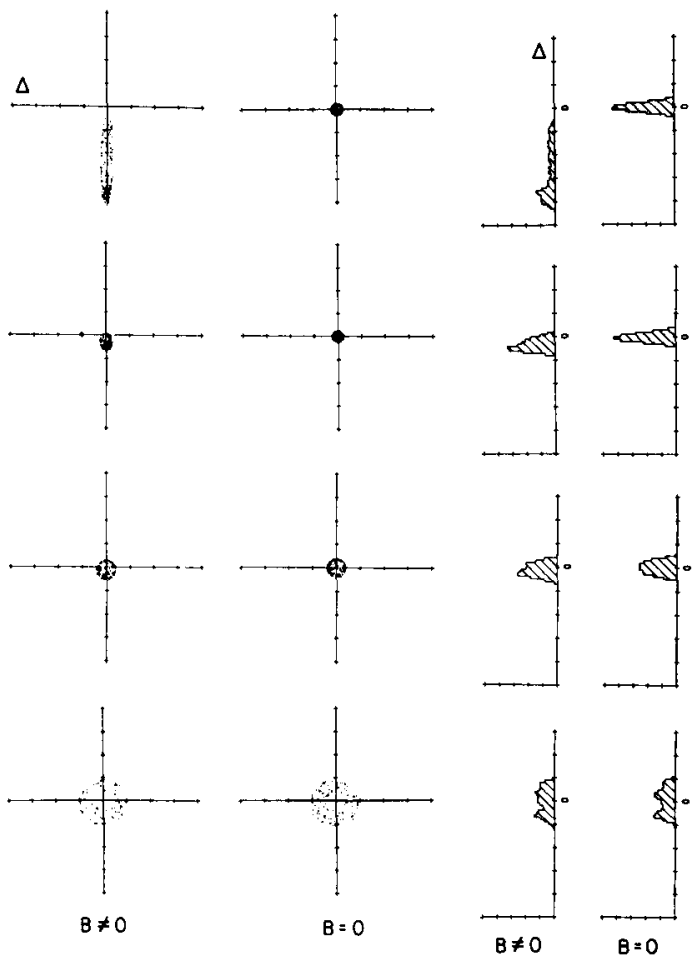

Fig. 7. (Left) Two panels showing the distribution of grains of various sizes (top to bottom, $0.03 \mu \mathrm{m}, 0.1 \mu \mathrm{m}, 0.3 \mu \mathrm{m}$, and $1.0 \mu \mathrm{m}$ ) in a plane normal to the Sun-comet axis of comet Giacobini-Zinner at a distance of $-10^{4} \mathrm{~km}$ behind the nucleus. The first column shows the distributions when the effects of the interplanetary magnetic field are included $(\vec{B} \neq \overrightarrow{0})$. (Right) Two panels showing histograms of the column densities (in arbitrary units) corresponding to the appropriate distributions on the left. The third column corresponds to the case $\vec{B} \neq \overrightarrow{0}$, while the fourth column corresponds to the case $\vec{B}=\overrightarrow{0}$. The unit length scale $(\Delta)$ in each case corresponds to $10^{5} \mathrm{~km}$, and the nucleus is always located at the origin of coordinates (from Horanyi and Mendis, 1986b).

The NASA International Comet Explorer (ICE) spacecraft intercepted the tail of comet Giacobini-Zinner on September 11, 1985, at a distance about $8 \times 10^{3} \mathrm{~km}$ from the nucleus, moving in a generally south to north direction in the comet's reference frame. 
Although the spacecraft did not carry a dust detector, the plasma wave instrument detected impulsive signals that were attributed to dust impacts on the spacecraft (Gurnett et al., 1986). While an asymmetry in the impact rate between the inbound and outbound legs, consistent with the prediction for charged grains was indeed observed, the asymmetry was rather small and may also be consistent with non-isotropic emission of grains from the nucleus, as suggested by Gurnett et al., (1986).

Horanyi and Mendis (1987) also considered the effect of a sector boundary crossing on the dust tail of a comet. When the grains are electrically charged, the traversal of the sector boundary along the tail leads to a reversal in the electrical acceleration on the grains due to the reversal of the convectional electric field. This leads to a wave-like feature in the distribution of the smaller $(\mathrm{a} \leq 0.3 \mu \mathrm{m})$ grains. These authors attribute the peculiar "wavy" morphology of the dust tail of comet Ikeya-Siki (1965f) to such an effect.

\section{Conclusions}

Electrostatic charging of cometary dust can also have physical effects on the grains such as electrostatic disruption. This question has been recently reviewed elsewhere (Mendis, 1989) and will not be further considered here. We merely state that the dust "packets," observed in the environment of comet Halley by dust detectors onboard VEGA-1 and VEGA-2 (Simpson et al., 1987) and attributed to the breakup of larger grains, are entirely consistent with electrostatic disruption. Indeed, only grain potentials $(|\phi|-10$ to $20 \mathrm{~V})$ need to be attained before such grains break up (Mendis and Horanyi, 1989). In this review, we have confined ourselves to a discussion of the dynamical consequences of electrostatic charging. While several observations are consistent with the anticipated consequences of grain charging, they are by no means conclusive. On the other hand, it is easy to see that even with the modest electric charges expected in the cometary environment, the electrodynamic forces experienced by the smallest grains in observed dust mass spectrum are larger than or comparable to the radiation pressure force. Consequently, these forces cannot be ignored in considering the dynamics of such grains.

\section{Acknowledgements}

We wish to acknowledge support the following grants: (M.H.) NAGW 1656 of the NASA Space Science Program, (D.A.M.) NAGW 1502 of the NASA Planetary Atmospheres Program, and NRA 88-055A-02 of the NASA Space Science Program.

\section{References}

Arrhenius, S. A., 1900, Uber die Ursache der Nordlichter, Phys. Zeitschr, 2, 81.

Bessel, F. W, 1836, Beobachtungen Uber die physische Beschaffenheit des Halley'schen Kometen und dadurch veranlasste Bemerkungen, Astron. Nachr., 13, 185.

Divine, N., 1984, Status of dust modelling for comet Halley, JPL interoffice memo; 5137-84 (internal document). 
Finson, M. L., and Probstein, R. F., 1968a, A theory of dust comets-I. Model and equations, Astrophys. J., 154, 327.

Finson, M. L., and Probstein R. F., 1968b, A theory of dust comets -II. Results for comet Arend-Roland, Astrophys. J., 154, 353.

Gombosi, T. J., and Horanyi, 1986, Time-dependent numerical modelling of dust halo formation at comets, Astrophys. J., 311, 491.

Hanner, M. S., 1980, Physical characteristics of cometary dust from optical studies, in Solid Particles in the Solar System (I. Halliday and B. A. McIntosh, Eds.), Dordrecht, Holland: D. Reidel Publ. Co., p. 233.

Horanyi, M., and Mendis, D.A., 1985, Trajectories of charged dust grains in the cometary environment, Astrophys. J., 294, 357.

Horanyi, M., and Mendis, D.A., 1986a, The effects of electrostatic charging on the dust distribution at Halley's comet, Astrophys. J., 307, 800.

Horanyi, M., and Mendis, D.A., 1986b, The dynamics of charged dust in the tail of comet Giacobini-Zinner, J. Geophys. Res., 91, 355.

Horanyi, M., and Mendis, D.A., 1987, The effect of a sector boundary crossing on the cometary dust tail, Earth, Moon, and Planets, 37, 71.

Mendis, D.A., Houpis, H.L.F., and Marconi, M.L., 1985, The physics of comets, Fund. Cosmic. Phys., 10, 1.

Mendis, D.A., 1989, Dust in the plasma environment of planets and comets, in Laboratory and Space Plasmas (H. Kikuchi, Ed.), Tokyo: Springer-Verlag, p. 51.

Mendis, D.A., and Horanyi, M.,1989, Dust-plasma interactions in the cometary environment, paper read at the Chapman Conference: Cometary Plasma Processes (Guildford, England, July 1989. To appear in the Proceedings (A. Johnstone, Ed.).

Notni, P., 1966, On the forces acting an charged dust particles in cometary atmospheres, in Nature et Origine des Cométes, Mem. Soc. R. Sci. Liege., 12, (Ser 5), 379.

Notni, P. and Tiersch, H., 1987, Charging of dust particles in comets and in interplanetary space, Astron. Astrophys., 187, 796.

Probstein, R. F., 1968, The dusty gas dynamics of comet heads, in Problems of hydrodynamics and continuum mechanics, Soc. Industr. Appl. Math, 568.

Rahe, J., Donn, B., and Wurm, K., 1969, Atlas of Cometary Forms, NASA SP-198, p. 61.

Schmidt, H.U., and Wegmann, R., 1982, Plasma flow and magnetic fields, in Comets (L.L. Wilkening, Ed.), Tucson: Univ. of Arizona Press, p. 538.

Schwarzchild L., 1901, Der druck des Lichtes auf kleine Kuglen und die Arrhenius'sche. Theorie des Cometenschweife, Sitz. Bayer. Acad. Wiss. Munchen, 1901, 293.

Sekanina, Z., 1987, Anisotropic emission from comets: Fans versus jets. 1. Concept and modelling, Symp. on Diversity and Similarity of Comets, ESA SP-273, p. 315.

Simpson, J.A., Rabinowitz, D., Tuzzolino, A.J., Ksanfomality, L.V., and Sagdeev, R.Z., 1987, The dust coma of comet P/Halley: measurements on Vega-1 and Vega-2 spacecraft, 1987, Astron. Astrophys., 187, 742. 
Vaisberg, O.L., Smirnov, V., Omelchenko, A., Gorn L., and Iovlev, M., 1987, Spatial and mass distribution of low mass dust particles $\left(\mathrm{m}<10^{-10} \mathrm{~g}\right)$ in comet P/Halley's coma, Astron. Astrophys., 187, 753.

Wallis, M.K., and Hassan, M.H.A., 1983, Electrodynamics of submicron dust in the cometary coma, Astron. Astrophys., 12, 10.

Whipple, E. C., 1981, Potentials of surfaces in space, Rep. Prog. Phys., 44, 1197. 